\title{
The Three R's of Computer Vision: Recognition, Reconstruction and Reorganization
}

\author{
Jitendra Malik ${ }^{\mathrm{a}, * *}$, Pablo Arbeláez ${ }^{\mathrm{b}}$, João Carreira ${ }^{\mathrm{a}}$, Katerina Fragkiadaki ${ }^{\mathrm{a}}$, Ross Girshick ${ }^{\mathrm{c}}$, Georgia Gkioxaria ${ }^{\mathrm{a}}$, Saurabh Gupta ${ }^{\mathrm{a}}$, \\ Bharath Hariharan ${ }^{\mathrm{c}}$, Abhishek Kar ${ }^{\mathrm{a}}$, Shubham Tulsiani ${ }^{\mathrm{a}}$ \\ ${ }^{a}$ EECS, UC Berkeley, Berkeley, CA 94720, USA \\ ${ }^{b}$ Universidad de los Andes, Bogotá 111711, Colombia \\ ${ }^{c}$ Facebook, Seattle WA 98101, USA
}

\begin{abstract}
We argue for the importance of the interaction between recognition, reconstruction and re-organization, and propose that as a unifying framework for computer vision. In this view, recognition of objects is reciprocally linked to re-organization, with bottom-up grouping processes generating candidates, which can be classified using top down knowledge, following which the segmentations can be refined again. Recognition of 3D objects could benefit from a reconstruction of 3D structure, and 3D reconstruction can benefit from object category-specific priors. We also show that reconstruction of 3D structure from video data goes hand in hand with the reorganization of the scene. We demonstrate pipelined versions of two systems, one for RGB-D images, and another for RGB images, which produce rich 3D scene interpretations in this framework.
\end{abstract}

(c) 2016 Elsevier Ltd. All rights reserved.

\section{Introduction}

The central problems in computer vision are recognition, reconstruction and reorganization.

Recognition is about attaching semantic category labels to objects and scenes as well as to events and activities. Part-whole hierarchies (partonomies) as well as categorysubcategory hierarchies (taxonomies) are aspects of recognition. Fine-grained category recognition includes as an extreme case instance level identification (e.g. Barack Obama's face).

Reconstruction is traditionally about recovering threedimensional geometry of the world from one or more of its images. We interpret the term more broadly as "inverse graphics" - estimating shape, spatial layout, reflectance and illumination - which could be used together to render the scene to produce an image.

Reorganization is our term for what is usually called "perceptual organization" in human vision; the "re" prefix makes the analogy with recognition and reconstruction more salient. In computer vision the terms grouping and segmentation are used with approximately the same general meaning.

\footnotetext{
${ }^{* *}$ Corresponding author: Tel.: +1-510-642-7597

e-mail: malik@eecs. berkeley.edu (Jitendra Malik)
}

Mathematical modeling of the fundamental problems of vision can be traced back to geometers such as Euclid (Burton, 1945), scientists such as Helmholtz (1925), and photogrammetrists such as Kruppa (1913). In the twentieth century, the Gestaltists led by Wertheimer (1923) emphasized the importance of perceptual organization. Gibson (1950) pointed out the many cues which enable a moving observer to perceive the three-dimensional structure of the visual world.

The advent of computers in the middle of the twentieth century meant that one could now develop algorithms for various vision tasks and test them on images, thus creating the field of computer vision. Roberts (1963) is often cited as the first paper in this field, though there was work on image processing and pattern recognition even before that. In recent years, progress has been very rapid, aided not only by fast computers, but also large annotated image collection such as ImageNet (Deng et al., 2009).

But is there a unifying framework for the field of computer vision? If one looks at the proceedings of a recent computer vision conference, one would notice a variety of applications using a wide range of techniques such as convex optimization, geometry, probabilistic graphical models, neural networks, and image processing. In the early days of computational vision, in the 1970s and 1980s, there was a broad agreement that vision could be usefully broken up into the stages of low level, 
Fig. 1. The 3R's of vision: Recognition, Reconstruction and Reorganization. Each of the six directed arcs in this figure is a useful direction of information flow.

\section{The 3 R's of Computer Vision}

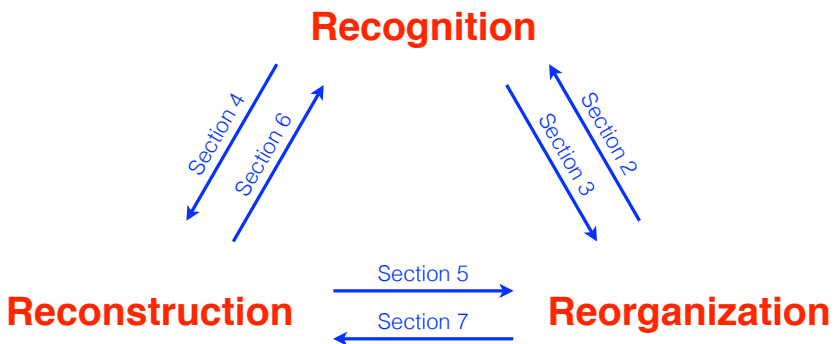

mid level and high level vision. Marr (1982) is perhaps the best articulation of this point of view, with low level vision corresponding to processes such as edge detection, mid level vision leading to representation of surfaces, and high level vision corresponding to object recognition. The process was thought of a being primarily feed-forward and bottom up. In the 1990s, this consensus gradually dissipated. Shape-from-X modules, with the exception of those based on multiple view geometry, proved to be not robust for general images, so the bottom up construction of Marr's desired 2.5D sketch proved infeasible. On the other hand, machine learning approaches to object recognition based on sliding windows started to succeed on real world images e.g. Viola and Jones (2001)'s work on face detection, and these didn't quite fit Marr's paradigm.

Back in the 1990s, one of us, Malik (2001) argued that grouping and recognition ought to be considered together. Bottom up grouping could produce candidates for consideration by a recognition module. Another paper from our group, Ren and Malik (2003) advocated the use of superpixels for a variety of tasks. This got some traction e.g. multiple segmentation hypotheses were used by Hoiem et al. (2005) to estimate the rough geometric scene structure and by Russell et al. (2006) to automatically discover object classes in a set of images, and $(\mathrm{Gu}$ et al., 2009) showed what were then state of the art results on the ETH-Z dataset. But the dominant paradigm remained that of sliding windows, and the state of the art algorithms on the PASCAL VOC challenge through 2011 were in that paradigm.

This has changed. The "selective search" algorithm of Uijlings et al. (2013) popularized the multiple segmentation approach for object detection by showing strong results on PASCAL object detection. EdgeBoxes (Zitnick and Dollár, 2014) outputs high-quality rectangular (box) proposals quickly $(\sim 0.3 \mathrm{~s}$ per image). Other methods focus on pixel-wise segmentation, producing regions instead of boxes. Top performing approaches include CPMC (Carreira and Sminchisescu, 2012), RIGOR (Humayun et al., 2014), MCG (Arbeláez et al., 2014), and GOP (Krähenbühl and Koltun, 2014). For a more in-depth survey of proposal algorithms, Hosang et al. (2015) provide an insightful meta-evaluation of recent methods.

In this paper we propose to go much further than the link between recognition and reorganization. That could be done with purely 2D reasoning, but surely our final percept must incorporate the $3 \mathrm{D}$ nature of the world? We will highlight a point of view that one of us (Malik) has been advocating for several years now, that instead of the classical separation of vision into low level, mid level and high level vision, it is more fruitful to think of vision as resulting from the interaction of three processes: recognition, reconstruction and reorganization which operate in tandem, and where each provides input to the others and fruitfully exploits their output. We aim for a grand unified theory of these processes, but in the immediate future it may be best to model various pairwise interactions, giving us insight into the representations that prove most productive and useful. In the next six sections, we present case studies which make this point, and we conclude with a pipeline which puts the different stages together.

Note that the emphasis of this paper is on the relationship between the 3R's of vision, which is somewhat independent of the (very important) choice of features needed to implement particular algorithms. During the 1970s and 1980s, the practice of computer vision was dominated by features such as edges and corners which offered the benefit of massive data compression, a necessity in a time when computing power was orders of magnitude less than today. The community moved on to the use of linear filters such as Gaussian derivatives, Gabors and Haar wavelets in the 1990s. The next big change was the widespread use of histogram based features such as SIFT (Lowe, 2004) and HOG (Dalal and Triggs, 2005). While these dominated for more than a decade, we are now completing yet another transition, that to "emergent" features from the top layers of a multilayer convolutional neural network (LeCun et al., 1989) trained in a supervised fashion on a large image classification task. Neural networks have proved very compatible to the synthesis of recognition, reconstruction and reorganization.

\section{Reorganization helps recognition}

As noted earlier, the dominant approach to object detection has been based on sliding-window detectors. This approach goes back (at least) to early face detectors (Vaillant et al., 1994), and continued with HOG-based pedestrian detection (Dalal and Triggs, 2005), and part-based generic object detection (Felzenszwalb et al., 2010). Straightforward application requires all objects to share a common aspect ratio. The aspect ratio problem can be addressed with mixture models (e.g., (Felzenszwalb et al., 2010)), where each component specializes in a narrow band of aspect ratios, or with bounding-box regression (e.g., (Felzenszwalb et al., 2010; Sermanet et al., 2014)).

The alternative is to first compute a pool of (likely overlapping) image regions, each one serving as a candidate object, and then to filter these candidates in a way that aims to retain only the true objects. By combining this idea with the use of convolutional network features, pretrained on an auxiliary task of classifying Imagenet, we get the Region-based Convolutional Network (R-CNN) which we describe next.

At test time, R-CNN generates around 2000 categoryindependent region proposals for the input image, extracts a fixed-length feature vector from each proposal using a convolutional neural network (CNN) (LeCun et al., 1989), and then 
Fig. 2. R-CNN: Region-based Convolutional Network: Object detection system overview. Our system (1) takes an input image, (2) extracts around 2000 bottom-up region proposals, (3) computes features for each proposal using a large convolutional network (CNN), and then (4) classifies each region using class- specific linear SVMs. We trained an R-CNN that achieves a mean average precision (mAP) of $62.9 \%$ on PASCAL VOC 2010. For comparison, Uijlings et al. (2013) report $35.1 \% \mathrm{mAP}$ using the same region proposals, but with a spatial pyramid and bag-of-visual-words approach. The popular deformable part models perform at $33.4 \%$. On the 200-class ILSVRC2013 detection dataset, we trained an R-CNN with a mAP of 31.4\%, a large improvement over OverFeat (Sermanet et al., 2014), which had the previous best result at $24.3 \% \mathrm{mAP}$.

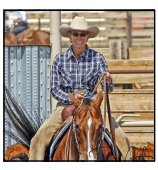

1. Input image

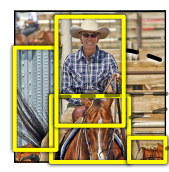

2. Extract region proposals $(\sim 2 \mathrm{k})$

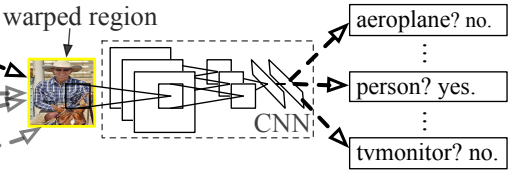

3. Compute $\mathrm{CNN}$ features
4. Classify regions classifies each region with category-specific linear SVMs. We use a simple warping technique (anisotropic image scaling) to compute a fixed-size CNN input from each region proposal, regardless of the region's shape. Figure 2 shows an overview of a Region-based Convolutional Network (R-CNN) and Table 1 presents some of our results.

R-CNNs scale very well with the number of object classes to detect because nearly all computation is shared between all object categories. The only class-specific computations are a reasonably small matrix-vector product and greedy non-maximum suppression. Although these computations scale linearly with the number of categories, the scale factor is small. Measured empirically, it takes only $30 \mathrm{~ms}$ longer to detect 200 classes than 20 classes on a CPU, without any approximations. This makes it feasible to rapidly detect tens of thousands of object categories without any modifications to the core algorithm.

Despite this graceful scaling behavior, an R-CNN can take 10 to 45 seconds per image on a GPU, depending on the network used, since each region is passed through the network independently. Recent work from He et al. (2014) ("SPPnet") improves R-CNN efficiency by sharing computation through a feature pyramid, allowing for detection at a few frames per second. Building on SPPnet, Girshick (2015) shows that it is possible to further reduce training and testing times, while improving detection accuracy and simplifying the training process, using an approach called "Fast R-CNN." Fast R-CNN reduces testing times to 50 to $300 \mathrm{~ms}$ per image, depending on network architecture.

Turning to the task of action recognition, R-CNN can be adapted to detect actions in space and time efficiently with videos considered as input, as we show in Gkioxari and Malik (2015).

Most work in the field (Poppe, 2010; Weinland et al., 2011; Aggarwal and Ryoo, 2011) has focused on the task of action classification, i.e. "Is an action present in the video?" The state-of-the-art approach in this field by Wang and Schmid (2013) uses dense point trajectories, where features are extracted from regions tracked using optical flow. More recently, Simonyan and Zisserman (2014a) use two-stream CNNs to
Fig. 3. Finding Action Tubes: Our basic model for action detection. We use action specific SVM classifiers on spatio-temporal features. The features are extracted from the fc7 layer of two CNNs, spatial-CNN and motion$\mathrm{CNN}$, which were trained to detect actions using static and motion cues, respectively.

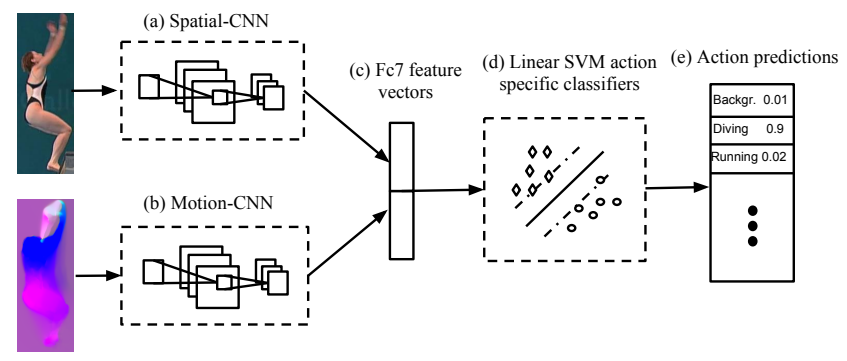

classify videos. The first CNN operates on the RGB frames, while the second CNN on the corresponding optical flow. They weight the predictions from the two CNNs and average the scores across frames to make the final prediction for the whole video. On the other hand, the task of action detection, i.e. "Is there an action and where is it in the video?" is less studied despite its higher potential for practical applications.

Inspired by the recent progress in object detection, we build models which localize and classify actions in video in both space and time. We call our predictions Action Tubes. Figure 3 shows the design of our action models. We start from bottom-up region proposals, similar to R-CNN. We train two CNNs, one on the RGB signal of the region (spatial-CNN) and one on the corresponding optical flow signal (motion-CNN). We combine the fc7 features of the two networks to train SVM classifiers to discriminate among the actions and the background. To produce temporarily coherent predictions, Action Tubes, we link action detections in time.

As mentioned earlier, R-CNN can be quite slow since each region is processed independently. One way around it is to share computation between regions. In the case of video, the motion signal is a strong cue and can be used to extract salient regions. Indeed if regions which don't contain enough motion are discarded, $85 \%$ of the computation can be saved with a loss of only $4 \%$ in recall.

Our detection pipeline makes predictions at every frame. Our goal is to align those predictions in time to produce detection tubes which follow the action across frames. For linking we use the spatial overlap of predictions in consecutive frames as well as their confidence scores. We formulate the problem using dynamic programming and infer the regions which comprise the Action Tubes. Figure 4 shows examples of action tubes on J-HMDB (Jhuang et al., 2013).

Quantitatively, Table 2 shows the performance of Action Tubes on J-HMDB. The combination of the spatial- and motion-CNN performs significantly better compared to the single CNNs, showing the significance of static and motion cues for the task of action recognition. On UCF Sports (Rodriguez et al., 2008), we compare Action Tubes with other approaches. Figure 5 shows AUC for various values of intersection-overunion, which define the threshold of spatial overlap with the ground truth for a correct prediction. 
Table 1. Detection average precision (\%) on VOC 2010 test. AlexNet is the CNN architecture from Krizhevsky et al. (2012) and VGG16 from Simonyan and Zisserman (2014b). R-CNNs are most directly comparable to UVA and Regionlets since all methods use selective search region proposals. Bounding-box regression (BB) is described in (Girshick et al., 2015). DPM and SegDPM use context rescoring not used by the other methods. SegDPM and all R-CNNs use additional training data.

\begin{tabular}{|c|c|c|c|c|c|c|c|c|c|c|c|c|c|c|c|c|c|c|c|c|c|}
\hline VOC 2010 test & aero & bike & bird & boat & bottle & bus & car & at & hair & cow & ble & dog & rs & e & pe & plant & eep & sofa & train & tv & $\mathrm{mAP}$ \\
\hline DPM v5 (Girshick et al.) & 49.2 & 53.8 & 13.1 & 15.3 & 35.5 & 53.4 & 49.7 & 27.0 & 17.2 & 28.8 & 14.7 & 17.8 & 46.4 & 51.2 & 47.7 & 10.8 & 34.2 & 20.7 & 43.8 & 38.3 & 33.4 \\
\hline Regionlets (Wang et al., 2013) & 65.0 & 48.9 & 25.9 & 24.6 & 24.5 & 56.1 & 54.5 & 51.2 & 17.0 & 28.9 & 30.2 & 35.8 & 40.2 & 55.7 & 43.5 & 14.3 & 43.9 & 32.6 & 54.0 & 45.9 & 39.7 \\
\hline R-CNN AlexNet & 67.1 & 64.1 & 46.7 & 32.0 & 30.5 & 56.4 & 57.2 & 65.9 & 27.0 & 47.3 & 40.9 & 66.6 & 57.8 & 65.9 & 53.6 & 26.7 & 56.5 & 38.1 & 52.8 & 50.2 & 50.2 \\
\hline R-CNN AlexNet BB & 71.8 & 65.8 & 53.0 & 36.8 & 35.9 & 59.7 & 60.0 & 69.9 & 27.9 & 50.6 & 41.4 & 70.0 & 62.0 & 69.0 & 58.1 & 29.5 & 59.4 & 39.3 & 61.2 & 52.4 & 53.7 \\
\hline $\mathrm{R}-\mathrm{CNN}$ & 76.5 & 70.4 & 58.0 & 40.2 & 39.6 & 61.8 & 63.7 & 81.0 & 36.2 & 64.5 & 45.7 & 80.5 & 71.9 & 74.3 & 60.6 & 31.5 & 64.7 & 52.5 & 64.6 & 57.2 & 59.8 \\
\hline
\end{tabular}

Table 2. Results and ablation study on J-HMDB (averaged over the three splits). We report $A P$ for the spatial and motion component and their combination (full). The combination of the spatial- and motion-CNN performs significantly better, showing the significance of static and motion cues for the task of action recognition.

\begin{tabular}{|c|c|c|c|c|c|c|c|c|c|c|c|c|c|c|c|c|c|c|c|c|c|c|}
\hline $\mathbf{A P}(\%)$ & 氠 & $\frac{\bar{v}}{\tilde{J}}$ & 单 & 高吉 & 窝 & 音 & 营 $\bar{\Xi}$ & $\frac{\breve{c}}{\ddot{z}}$ & 言 & $\frac{\mathrm{g}}{\bar{z}}$ & $\begin{array}{l}\bar{E} \\
\bar{z} \\
\bar{z}\end{array}$ & $\Xi$ & 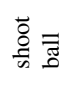 & ㅎํ요 & 蒿志 & : & 氶 & 点离 & 总 & 剪 & 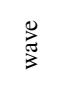 & $\begin{array}{l}\text { ⿷匚ँ } \\
\text { घ }\end{array}$ \\
\hline spatial-CNN & 67.1 & 34.4 & 37.2 & 36.3 & 93.8 & 7.3 & 14.4 & 29.6 & 80.2 & 93.9 & 17.4 & 10.0 & 8.8 & 71.2 & 45.8 & 17.7 & 11.6 & 38.5 & 20.4 & 40.5 & 19.4 & 37.9 \\
\hline full & 79.1 & 33.4 & 53.9 & 60.3 & 99.3 & 18.4 & 26.2 & 42.0 & 92.8 & 98.1 & 29.6 & 24.6 & 13.7 & 92.9 & 42.3 & 67.2 & 57.6 & 66.5 & 27.9 & 58.9 & 35.8 & 53.3 \\
\hline
\end{tabular}

Fig. 4. Results of Action Tubes on J-HMDB. We show the regions with red. The predicted action label is overlaid.

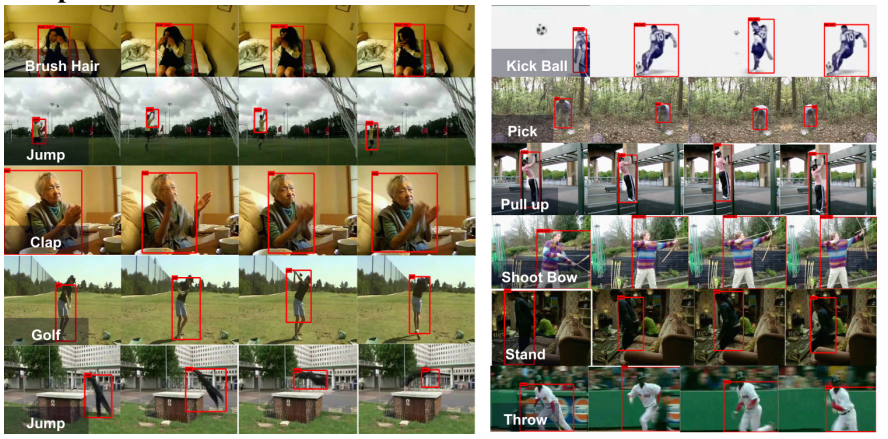

Finally, we demonstrate that Action Tubes improve action classification compared to the holistic approach, where spatialCNN is trained on the RGB frames and motion-CNN on the optical flow, similar to (Simonyan and Zisserman, 2014a). On J-HMDB, the holistic approach achieves an accuracy of $56.5 \%$ while Action Tubes yield $62.5 \%$. This shows that focusing on the actor is of great importance both for the case of action detection and action classification.

\section{Recognition helps reorganization}

Object proposal generation methods such as those described earlier typically rely on the coherence of color and texture to segment the image out into likely object candidates. However, such cues can often make mistakes. For instance, the boundary between the dog in Figure 6 and the wall is barely perceptible, while the meaningless contour between the dog's face and its torso is sharp. However, once an object detection approach such as R-CNN detects the dog, we can bring to bear our knowledge about dogs and segment out the dog much more
Fig. 5. AUC on UCF Sports for various values of intersection-over-union threshold of $\sigma$ ( $x$-axis). Red shows our approach. We consistently outperform other approaches by Jain et al. (2014), Wang et al. (2014) Tian et al. (2013) and Lan et al. (2011). The biggest improvement is being achieved at high values of overlap $(\sigma \geq 0.4)$.

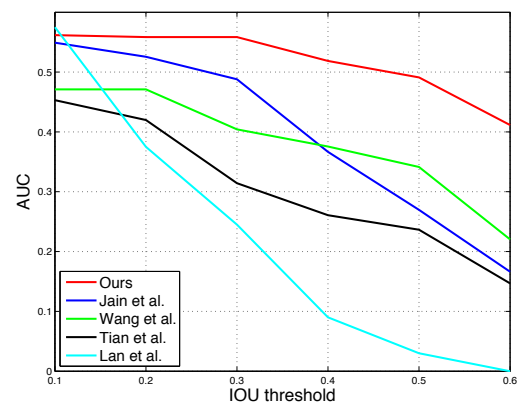

Fig. 6. An image of a dog (left), bottom-up contours (center) and segmentation predicted using top-down information (right).
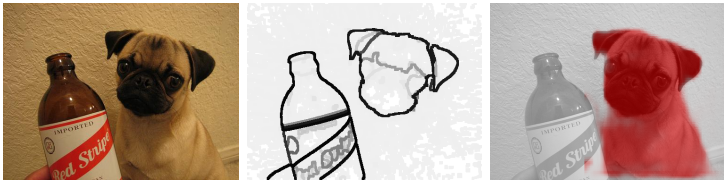

accurately. Recognition of the dog should thus aid us in accurate segmentation. See (Borenstein and Ullman, 2002) for an early implementation of this idea.

We explored this relationship between recognition and reorganization in two papers (Hariharan et al., 2014, 2015) where we presented the Simultaneous Detection and Segmentation (SDS) task: detect all instances of a category in an image and, for each instance, correctly mark the pixels that belong to it. Our algorithm for this task consists of the following steps: 
1. We use R-CNN (Section 2) to first detect objects of all categories along with coarse bounding boxes. We keep the non-maximum suppression threshold low to avoid prematurely removing any correct detection.

2. For each detected object, we make a top-down, categoryspecific prediction of the figure-ground.

3 . We then use the predicted segments to rescore the detections.

4. We finally run a round of non-max suppression to remove duplicate detections.

Next we describe how we predict the figure-ground mask for each detection and how we use the predicted segments to rescore the detections.

\subsection{Top-down prediction of figure-ground}

Given a detection, we predict a heatmap on the bounding box of the detection, which we splat onto the image. This heatmap encodes the probability that a particular location is inside the object. Thus, the task of predicting figure-ground masks reduces to classifying each location as being inside the object or not.

As in R-CNN, we use features from the CNN to do this classification. Typically, recognition algorithms such as R-CNN use the output of the last layer of the CNN. This makes sense when the task is assigning category labels to images or bounding boxes: the last layer is the most sensitive to category-level semantic information and the most invariant to "nuisance" variables such as pose, illumination, articulation, precise location and so on. However, when the task we are interested in is finergrained, as is the case in SDS, these nuisance variables are precisely what carries the signal. For such applications, the top layer is thus not the optimal representation.

The information that is generalized over in the top layer is present in intermediate layers, but intermediate layers are also much less sensitive to semantics. For instance, bar detectors in early layers might localize bars precisely but cannot discriminate between bars that are horse legs and bars that are tree trunks. This observation suggests that reasoning at multiple levels of abstraction and scale is necessary, mirroring other problems in computer vision (such as optical flow) where reasoning across multiple levels has proved beneficial.

To capture such reasoning, we developed the hypercolumn representation. We define the "hypercolumn" at a given input location as the outputs of all CNN units that lie above that location at all layers of the $\mathrm{CNN}$, stacked into one vector. (Because adjacent layers are correlated, in practice we need not consider all the layers but can simply sample a few.) Figure 7 shows a visualization of the idea. We borrow the term "hypercolumn" from neuroscience, where it is used to describe a set of V1 neurons sensitive to edges at multiple orientations and multiple frequencies arranged in a columnar structure (Hubel and Wiesel, 1962). The notion of combining predictions from multiple CNN layers is also described in (Sermanet et al., 2013; Long et al., 2015; Maire et al., 2014). There has also been prior work on semantic segmentation using CNNs (Farabet et al., 2013), though such work does not group pixels into individual instances.

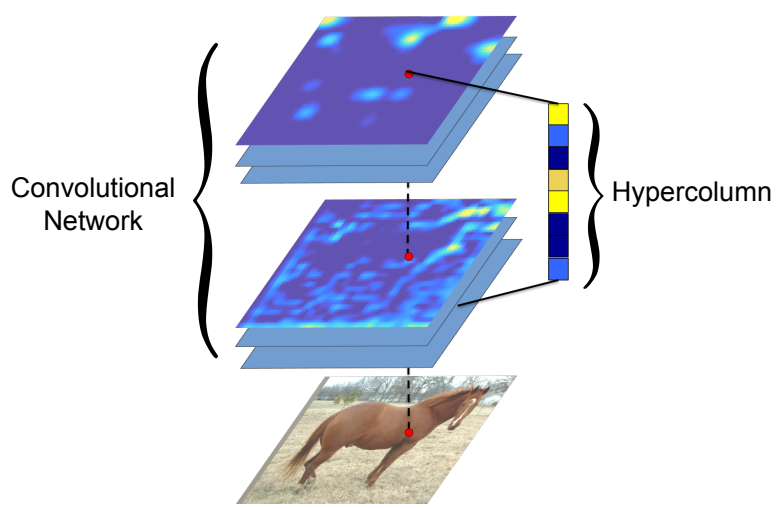

Fig. 7. The hypercolumn representation. The bottom image is the input, while the other images represent feature maps of different layers in the CNN. The hypercolumn at a pixel is the vector of activations of all units that lie above that pixel.

Fig. 8. The region classification network.

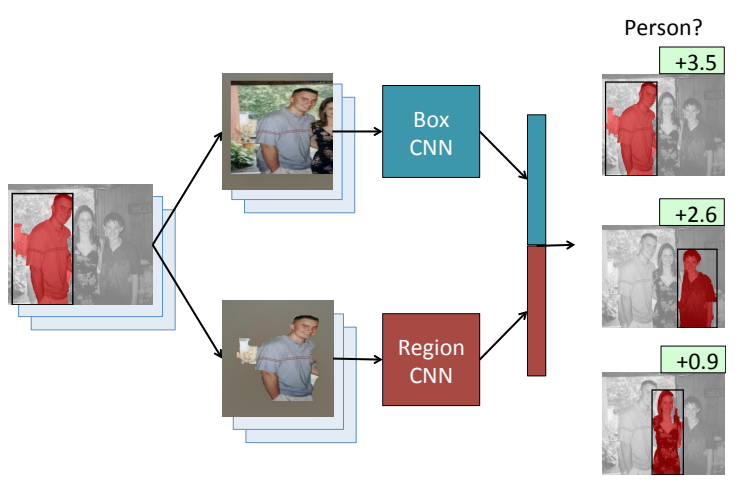

Given such a hypercolumn representation for each pixel, we can then use these as features to train pixel classifiers to label each pixel as figure-vs-ground. The full details of the implementation are in (Hariharan et al., 2015).

\subsection{Scoring region candidates}

Once we have produced a segmentation for each detection, we now rescore the detection. As for action recognition, we use a two-stream architecture as shown in Figure 8. The first stream operates on the cropped bounding box of the detection (the "box" pathway) while the second stream operates on the cropped bounding box with the predicted background masked (the "region" pathway). Features from the two streams are concatenated and passed into the classifier, and the entire network is trained jointly end-to-end.

\subsection{Evaluation metric}

We evaluate our performance using an extension of the bounding box detection metric (Hariharan et al., 2014; Tighe et al., 2014; Yang et al., 2012). The algorithm produces a ranked list of detections where each detection comes with a predicted segmentation. A detection is correct if its segmentation overlaps with the segmentation of a ground truth instance by more than a threshold. As in the classical bounding box task, 
Table 3. Performance of various systems on the SDS task.

\begin{tabular}{lccc}
\hline & Network & $\begin{array}{c}\mathrm{AP}^{r} \\
\text { at } 0.5\end{array}$ & $\begin{array}{c}\mathrm{AP}^{r} \\
\text { at } 0.7\end{array}$ \\
\hline Top layer only & AlexNet & 44.0 & 16.3 \\
Hypercolumn (Layers 7, 4, 2) & AlexNet & 49.1 & 29.1 \\
Hypercolumn (Layers 7, 4, 3) & VGG16 & 56.5 & 37.0 \\
+Rescore & VGG16 & 60.0 & 40.4 \\
\hline
\end{tabular}

Fig. 9. Qualitative results. In black is the bounding box and in red is the predicted segmentation.

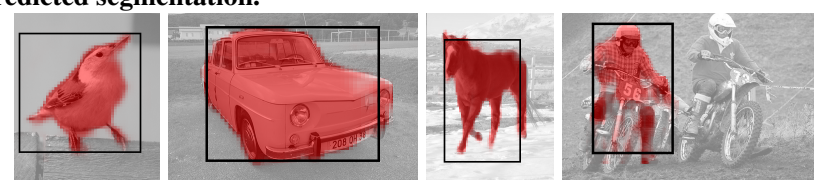

we penalize duplicates. With this labeling, we compute a precision recall (PR) curve, and the average precision (AP), which is the area under the curve. We call the AP computed in this way $\mathrm{AP}^{r}$. We report $\mathrm{AP}^{r}$ at overlap thresholds of 0.5 and 0.7 in Table 3.

We observe that:

1. Using three different layers to make the figure-ground prediction almost doubles $\mathrm{AP}^{r}$ at 0.7 , validating the intuition behind the hypercolumn representation.

2. Using a larger network leads to large performance gains (about 8 points).

3. Rescoring detections using segment-based features also provides a significant boost (about 3 points).

Qualitative segmentations are shown in Figure 9.

\section{Recognition helps reconstruction}

Consider Figure 10. As humans, we can easily perceive the 3D shape of the shown object, even though we might never have have seen this particular object instance. We can do this because we don't experience this image tabula rasa, but in the context of our "remembrance of things past". We can recognize that this is the image of a car as well as estimate the car's 3D pose. Previously seen cars enable us to develop a notion of the 3D shape of cars, which we can project to this particular instance using its determined pose. We also specialize our representation to this particular instance (e.g. any custom decorations it might have), signalling that both top-down and bottom-up cues influence our percept - see also (Nandakumar et al., 2011).

In order to operationalize these observations into an approach that can perceive the shape of novel instances we need recognition systems that can infer the location and pose of objects as well as category-specific shape models that capture knowledge about previously seen objects. We previously described a simultaneous detection and segmentation (SDS) system that can give us the instance segmentations of the objects present in the image. We now describe our pose (viewpoint) estimation, originally published in (Tulsiani and Malik, 2015), and categoryspecific shape modeling systems - from (Kar et al., 2015). We
Fig. 10. Automatic object reconstruction from a single image. Our method leverages estimated instance segmentations and predicted viewpoints to generate a full 3D mesh and high frequency 2.5D depth maps.

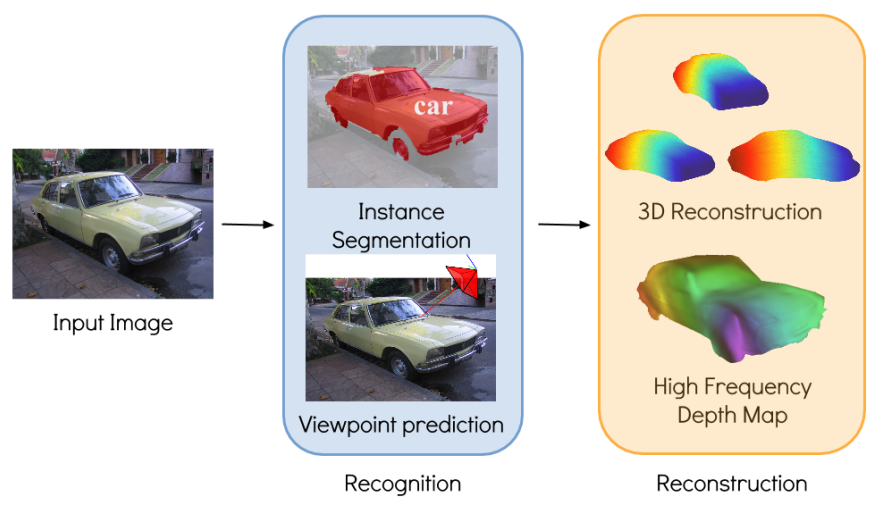

then show that building upon these object recognition and reconstruction systems, 3D object reconstruction from a single image in the wild can be readily addressed as shown in Fig. 10.

\subsection{Viewpoint Prediction}

We formulate the problem of viewpoint prediction as predicting three euler angles ( azimuth, elevation and cyclo-rotation) corresponding to the instance. We train a CNN based architecture which can implicitly capture and aggregate local evidences for predicting the euler angles to obtain a viewpoint estimate. Our CNN architecture additionally enables learning a shared feature representation across all categories.

We visualize the predictions of our approach in Figure 11. It can be seen that our predictions are accurate even for the $75^{\text {th }}$ percentile instances. To evaluate for the task of simultaneous detection and viewpoint estimation, we use the AVP metric introduced by Xiang et al. (2014). Each detection candidate has an associated viewpoint and the detection is labeled correct if it has a correct predicted viewpoint bin as well as a correct localization (bounding box IoU $>0.5$ ). As shown in Table 4, our approach significantly outperforms previous methods.

Table 4. Mean performance of our approach for simultaneous detection and viewpoint estimation.

\begin{tabular}{lcccc} 
& \multicolumn{4}{c}{$A V P$} \\
\hline Number of bins & $\mathbf{4}$ & $\mathbf{8}$ & $\mathbf{1 6}$ & $\mathbf{2 4}$ \\
\hline Xiang et al. (2014) & 19.5 & 18.7 & 15.6 & 12.1 \\
Pepik et al. (2012) & 23.8 & 21.5 & 17.3 & 13.6 \\
Ghodrati et al. (2014) & 24.1 & 22.3 & 17.3 & 13.7 \\
ours & $\mathbf{4 9 . 1}$ & $\mathbf{4 4 . 5}$ & $\mathbf{3 6 . 0}$ & $\mathbf{3 1 . 1}$ \\
\hline
\end{tabular}

\subsection{Category-Specific Shape Models}

Unlike prior work that assumes high quality detailed annotations (Carreira et al., 2015), we are interested in reconstructing objects "in the wild". At the core of our approach are deformable 3D models that can be learned from 2D annotations available in existing object detection datasets and can be driven by noisy automatic object segmentations. These allow us to 
Fig. 11. Viewpoint predictions for unoccluded groundtruth instances using our algorithm. The columns show 15th, 30th, 45th, 60th, 75th and 90th percentile instances respectively in terms of the error. We visualize the predictions by rendering a 3D model using our predicted viewpoint.
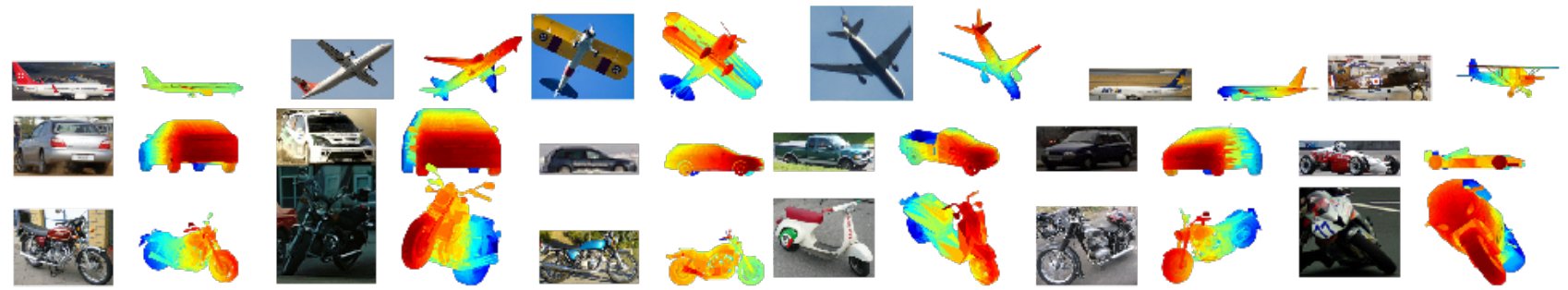

overcome the two main challenges to object reconstruction in the wild: 1) detection datasets typically have many classes and each may encompass wildly different shapes, making 3D model acquisition expensive. 2) 3D shape inference should be robust to any small imperfections that detectors produce, yet be expressive enough to represent rich shapes. We use the learned deformable 3D models to infer the shape for each detection in a novel input image and further complement it with a bottom-up module for recovering high-frequency shape details. The learning and inference steps are described below.

Fig. 12. Overview of our training pipeline for learning deformable shape models.

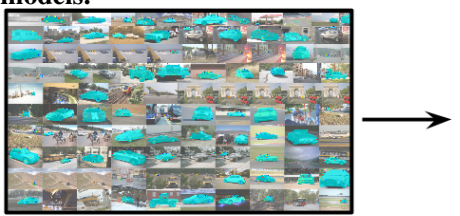

Annotated Image Collection

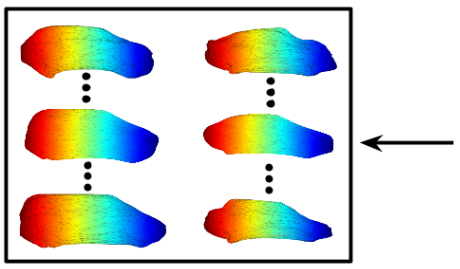

Mean Shapes and Deformation Modes

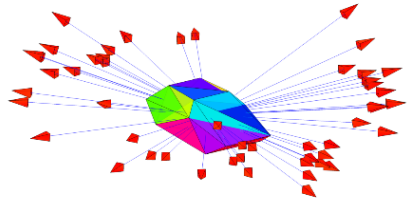

Camera Estimation

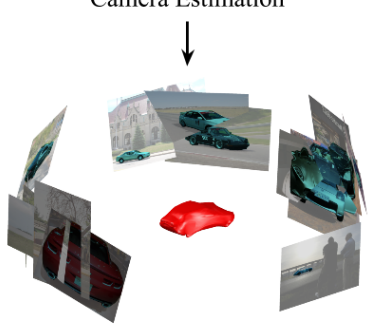

Learning 3D Shape Models from Silhouettes
Figure 12 illustrates our pipeline for learning shape models from just $2 \mathrm{D}$ training images, aided by ground truth segmentations and a few keypoints. We first use the NRSfM framework of Torresani et al. (2008), extended to incorporate silhouette information, to jointly estimate the camera viewpoints (rotation, translation and scale) for all training instances in each class.

We then learn the category level shape models by optimizing over a deformation basis of representative 3D shapes that best explain all silhouettes, conditioned on the camera viewpoints. We model our category shapes as deformable point clouds - one for each subcategory of the class. Our shape model $M=(\bar{S}, V)$ comprises of a mean shape $\bar{S}$ (learnt mean shapes for several classes are shown in Figure 13) and linear deformation bases $V=\left\{V_{1}, ., V_{K}\right\}$. We formulate our energy primarily based on image silhouettes and priors on natural shapes. These energies enforce that the shape for an instance is consistent with its silhouette $\left(E_{s}, E_{c}\right)$, shapes are locally consistent $\left(E_{l}\right)$, normals vary smoothly $\left(E_{n}\right)$ and the deformation parameters are small $\left(\left\|\alpha_{i k} V_{k}\right\|_{F}^{2}\right)$. Finally, we solve the constrained optimization in equation 1 using block-coordinate descent to learn the category level shape model. We refer the reader to (Kar et al., 2015) for detailed descriptions of the energy formulation and optimization.

$$
\begin{array}{ll}
\min _{\bar{S}, V, \alpha} & E_{l}(\bar{S}, V)+\sum_{i}\left(E_{s}^{i}+E_{k p}^{i}+E_{c}^{i}+E_{n}^{i}+\sum_{k}\left(\left\|\alpha_{i k} V_{k}\right\|_{F}^{2}\right)\right) \\
\text { subject to: } & S^{i}=\bar{S}+\sum_{k} \alpha_{i k} V_{k}
\end{array}
$$

Fig. 13. Mean shapes learnt for rigid classes in PASCAL VOC using our formulation. Color encodes depth when viewed frontally.

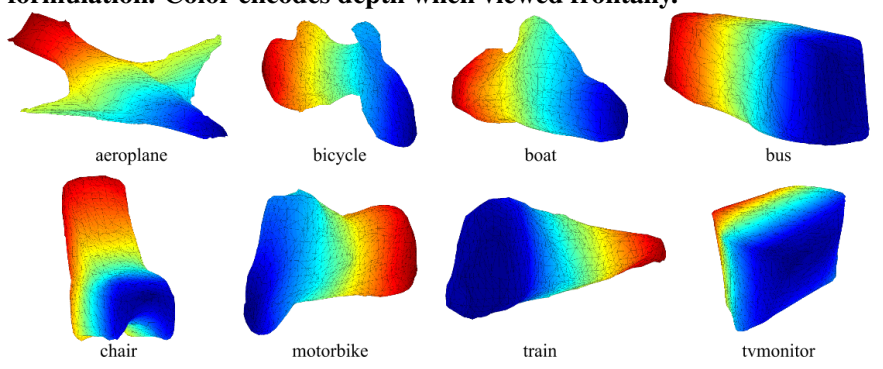

\section{Reconstruction helps reorganization}

RGB-D sensors like the Microsoft Kinect, provide a depth image in addition to the RGB image. We use this additional depth information as 'reconstruction' input and study the reorganization problems. In particular we study the problem of contour detection and region proposal generation.

\subsection{Contour Detection}

Depth images have $C^{0}$ and $C^{1}$ discontinuities corresponding to depth and surface normal edges in the scene. We design local gradient functions to capture both these sets of discontinuities. We call these gradients Depth Gradients $(D G)$ and Normal Gradients $N G$. We distinguish between convex and concave normal gradients $N G_{+}$and $N G_{-}$. This distinction is useful given concave normal gradient edges (such as those between the bed and the floor in Figure 14) are more likely to correspond to object 
Fig. 14. Contour Detection: Color image, depth image, contours from color image, contours from colour and depth image, contour labels.
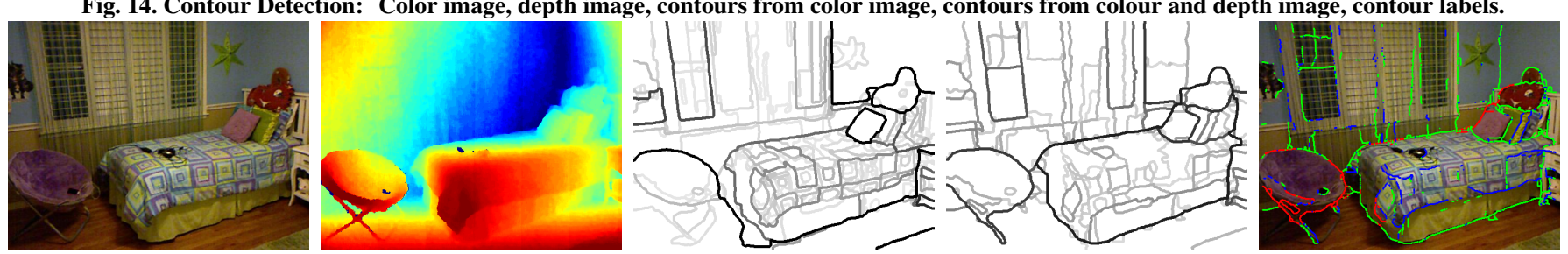

boundaries than convex gradient edges (such as those within the bed). We compute these gradients by estimating local planar approximations and computing the distance between these planes to compute $D G$ and the angle between them to estimate $N G_{+}$and $N G_{-}$.

We can then use this set of estimated gradients from depth images in existing contour detection systems for RGB and RGB-D images. We experimented with two such state-of-theart systems, RGB only gPb-ucm (Arbeláez et al., 2011) and RGB-D Structured Edges (Dollár and Zitnick, 2014) on the NYUD2 dataset (Silberman et al., 2012), and see improvements over both these methods. We show the precision recall curves for contour detection in Figure 15(left) and see that adding our gradients improves performance across the board (Gupta et al., 2014b, 2013).

Figure 14 shows visualizations for the obtained contours. As expected, we get less distracted with albedo, recall faint but important scene surface boundaries, weigh important object boundaries more, and get more complete objects out. We can also go a step further and label each boundary as being an occlusion boundary, convex normal edge (blue) or a concave normal edge(green).

\subsection{Region Proposals}

With this improved contour signal, we can generate region proposals for RGB-D images. For this we generalize MCG (Arbeláez et al., 2014) which is the state-of-the-art algorithm for generating region proposals on RGB images, to RGB-D images. MCG works by a) completing contours to form closed regions at multiple scales, b) combinatorially combining these closed regions to produce millions of candidates, and c) reranking these candidates to obtain a ranked list of about 2000 regions.

We generalize MCG in the following ways: a) we use our improved RGB-D contour signal from Section 5.1 and b) use features computed from the depth image for re-ranking the candidates. We measure the quality of our region proposals using standard region overlap metrics in Figure 15. We plot the number of regions on X-axis and the average best overlap on the Yaxis, and observe that with only 200 RGB-D region proposals per image, we can obtain the same average best overlap as one would get with 2000 regions using RGB based MCG (Gupta et al., 2014b), illustrating the quality of our contours and the utility of using reconstruction input in the form of depth images for the re-organization problem.
Fig. 15. Additional depth information from a Kinect like sensor improves the quality of bottom-up contours and region proposals.
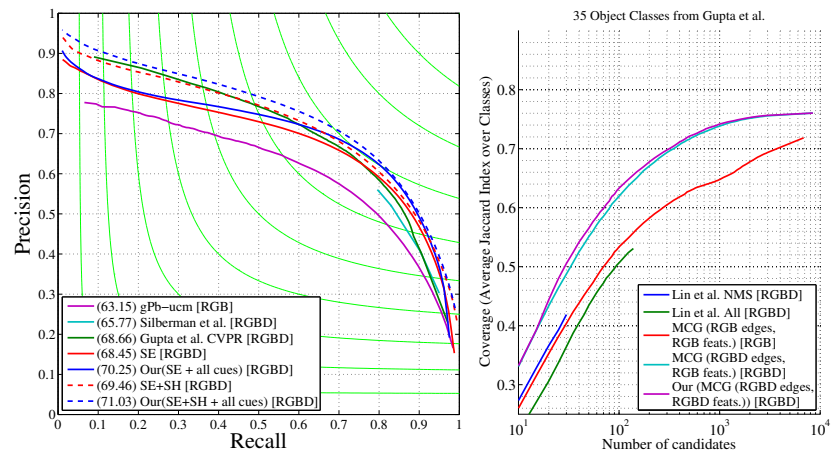

\section{Reconstruction helps recognition}

We can also use the depth image from a Kinect sensor to aid recognition. More specifically, we study how reconstruction input in the form of a depth image from a RGB-D sensor can be used to aid performance for the task of object detection.

We conduct these experiments on the NYUD2 dataset (Silberman et al., 2012). The set of categories that we study here included indoor furniture categories like chairs, beds, sofas, and tables.

We frame this problem as a feature learning problem and use a convolutional neural network to learn features from RGB-D images. To do this, we build on the R-CNN work (Girshick et al., 2014). We propose a novel geocentric embedding of depth images into horizontal disparity, height above ground and angle with gravity (denoted HHA) (we automatically estimate the direction of gravity (Gupta et al., 2013) and the height above ground from the depth image). This embedding captures basic physical properties that defines such object categories. For instance, consider a chair, it is characterized by a horizontal surface of a particular area at a particular height and a vertical surface of a particular area in a specific location relative to the horizontal surface. Our proposed embedding exposes this information to the feature learning algorithm.

In our experiments (reported in (Gupta et al., 2014b)), we observe the following: a) a CNN learned on color images (in our case a CNN (Krizhevsky et al., 2012) learned for image classification on ImageNet (Deng et al., 2009)) learns features which are general enough and transfer well to depth images, and b) using our proposed HHA embedding boosts performance over just using the depth image. Finally, when we combine our learned features on depth images with those coming from color images, we achieve state-of-the-art performance for ob- 
Table 5. Results for object detection on NYUD2 (Silberman et al., 2012): We report detection average precision, and compare against three baselines: RGB DPMs, RGBD-DPMs, and RGB R-CNN. For details refer to (Gupta et al., 2014b).

\begin{tabular}{|c|c|c|c|c|c|c|c|c|c|c|c|c|c|c|c|c|c|c|c|c|}
\hline & ฐ్ & 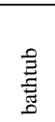 & $\ddot{D}$ & $\begin{array}{l}\frac{4}{2} \\
\frac{5}{5} \\
\frac{4}{0} \\
8 \\
8\end{array}$ & రั & 氠 & $\begin{array}{l}\text { 氙 } \\
\stackrel{\Xi}{0}\end{array}$ & $\begin{array}{l}\frac{y}{0} \\
\frac{\tilde{d}}{0}\end{array}$ & ఫ̊ & 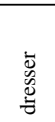 & 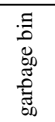 & 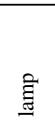 & 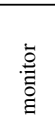 & 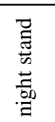 & $\stackrel{3}{\stackrel{3}{\Xi}}$ & 光 & ๘ँ & $\frac{0}{\frac{\pi}{0}}$ & $\begin{array}{l}\frac{\Xi}{0} \\
\frac{0}{3} \\
\frac{0}{2}\end{array}$ & $\frac{\overrightarrow{0}}{\vec{\theta}}$ \\
\hline RGB DPM & 9.0 & 0.9 & 27.6 & 9.0 & 0.1 & 7.8 & 7.3 & 0.7 & 2.5 & 1.4 & 6.6 & 22.2 & 10.0 & 9.2 & 4.3 & 5.9 & 9.4 & 5.5 & 5.8 & 34.4 \\
\hline RGBD-DPM & 23.9 & 19.3 & 56.0 & 17.5 & 0.6 & 23.5 & 24.0 & 6.2 & 9.5 & 16.4 & 26.7 & 26.7 & 34.9 & 32.6 & 20.7 & 22.8 & 34.2 & 17.2 & 19.5 & 45.1 \\
\hline RGB R-CNN & 22.5 & 16.9 & 45.3 & 28.5 & 0.7 & 25.9 & 30.4 & 9.7 & 16.3 & 18.9 & 15.7 & 27.9 & 32.5 & 17.0 & 11.1 & 16.6 & 29.4 & 12.7 & 27.4 & 44.1 \\
\hline Our & 37.3 & 44.4 & 71.0 & 32.9 & 1.4 & 43.3 & 44.0 & 15.1 & 24.5 & 30.4 & 39.4 & 36.5 & 52.6 & 40.0 & 34.8 & 36.1 & 53.9 & 24.4 & 37.5 & 46.8 \\
\hline
\end{tabular}

ject detection (Gupta et al., 2014b) (Table 5). To summarize the results briefly, RGB based Deformable Part Models (DPM) (Felzenszwalb et al., 2010) (long standing state-of-the-art detection method) give a mean average precision of $9.0 \%$, RGBD DPMs (Gupta et al., 2014a) boost performance to $23.9 \%$, current state-of-the-art RGB detection method R-CNN gives $22.5 \%$. In contrast, our method which effectively learns features from depth images, achieves a mean average precision of $37.3 \%$. The performance for the challenging chair category is at $43.3 \%$, up from $7.8 \%$ a couple of years ago, taking perception a step closer to being useful for robotics.

\section{Reorganization helps reconstruction}

Consider Fig. 16. The leftmost column depicts a video scene that contains multiple moving objects. We want to reconstruct their spatio-temporal 3D shapes. Extensive literature exists on reconstructing static scenes from monocular uncalibrated videos, a task also known as rigid Structure-from-Motion (SfM). Some works employ scaled orthographic cameras with rank shape priors, such as the seminal factorization work of Tomasi and Kanade (1991), while others assume perspective cameras and make use of epipolar constraints (Pollefeys et al., 1998). The case of deforming objects (a.k.a. Non-Rigid Structure-from-Motion (NRSfM)) has attracted less attention. Most NRSfM approaches assume as input pre-segmented, fulllength point trajectories extracted from the deforming surface (Bregler et al., 2000; Hartley and Vidal, 2008; Akhter et al., 2011; Dai et al., 2012; Garg et al., 2013).

In our work (Fragkiadaki et al., 2014), we jointly study video segmentation and 3D reconstruction of deforming objects and present a multiple hypotheses approach that deals with inaccurate video segments and temporally incomplete point tracks. For each object, our input is a trajectory matrix that holds $x, y$ pixel coordinates of the object's 2D point trajectories. Our output is a $3 \mathrm{D}$ trajectory matrix with the corresponding $X, Y, Z$ point coordinates, and a camera pose matrix that holds the per frame camera rotations. We assume scaled orthographic cameras which means that camera rotations correspond to truncated rotation matrices. Extracting 3D shape from monocular input is an under-constrained problem since many combinations of 3D point clouds and camera viewpoints give rise to the same 2D image projections. We use low-rank 3D shape priors to fight this ambiguity, similar to previous works (Bregler et al., 2000).
An overview of our approach is presented in Figure 16. Given a video sequence, we compute dense optical flow point trajectories and cluster them using normalized cuts on 2D motion similarities. We compute multiple trajectory clusterings to deal with segmentation ambiguities. In each cluster, 2D trajectories will be temporally incomplete, Fig. 16 3rd column shows in green the present and in red the missing trajectory entries for the belly dancer trajectory cluster. For each trajectory cluster, we first complete the $2 \mathrm{D}$ trajectory matrix using low-rank matrix completion, as in (Cabral et al., 2013; Burer and Monteiro, 2005). We then recover the camera poses through a rank 3 factorization and Euclidean upgrade of the camera pose matrix, as in (Tomasi and Kanade, 1991). Last, keeping the camera poses fixed, we minimize the reprojection error of the observed trajectory entries along with the nuclear norm of the 3D shape, similar to (Dai et al., 2012), using the nuclear norm regularized least squares algorithm of Toh and Yun (2010).

Reconstruction needs to be robust to segmentation mistakes. Motion trajectory clusters are inevitably polluted with "bleeding" trajectories that, although they reside on the background, anchor on occluding contours. We use morphological operations to discard such trajectories that do not belong to the shape subspace and confuse reconstruction.

A byproduct of our NRSfM algorithm is trajectory completion. The recovered 3D time-varying shape is backprojected in the image and the resulting 2D trajectories are completed through deformations, occlusions or other tracking ambiguities, such as lack of texture.

Figure 17 presents reconstruction results of our approach in videos from two popular video segmentation benchmarks, VSB100 (Galasso et al., 2013) and Moseg (Brox and Malik, 2010), that contain videos from Hollywood movies and Youtube. For each example we show a) the trajectory cluster, b) the present and missing trajectory points, and c) the depths of the visible (as estimated from ray casting) points, where red and blue denote close and far respectively. Our work was the first to show dense non-rigid reconstructions of objects from real videos, without employing object-specific shape priors.

\section{Putting it Together}

In our recent work (Gupta et al., 2015), we have brought concepts presented in this paper together to automatically being able to represent objects in RGB-D images with corresponding models from a 3D CAD model library. We achieve this using 
Fig. 16. Segmentation-based non-rigid structure from motion. Occluded trajectories on the belly dancer, that reside beyond the image border, are completed.
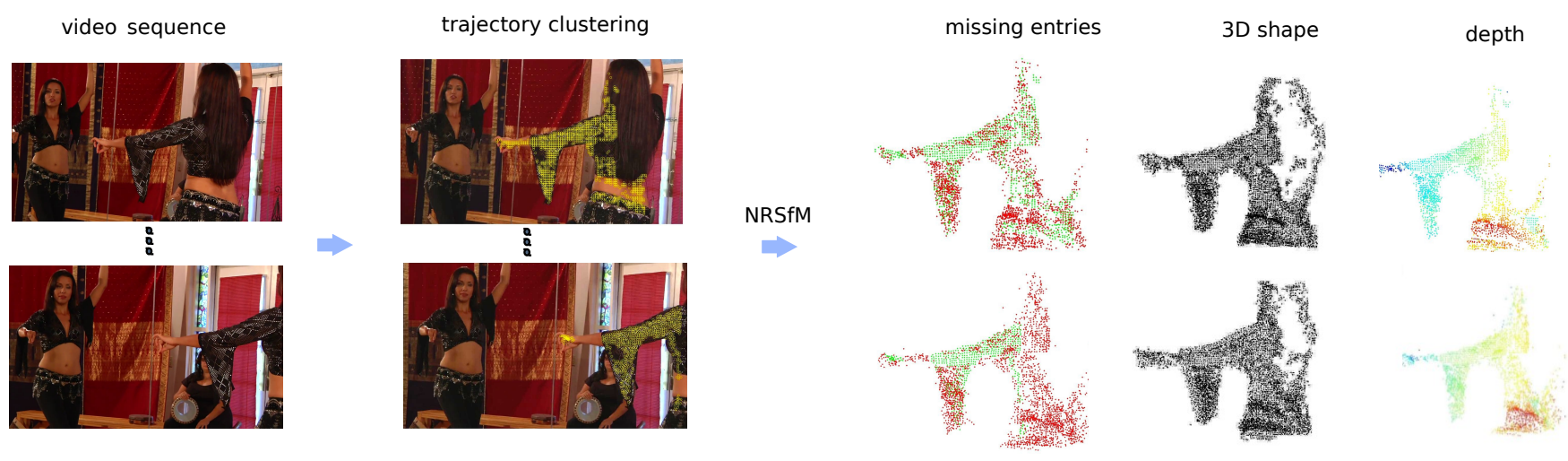

Fig. 17. Reconstruction results on the VSB100 and Moseg video segmentation benchmarks. We show in green and red present and missing trajectory entries, respectively. In the depth image red is close, blue is far.
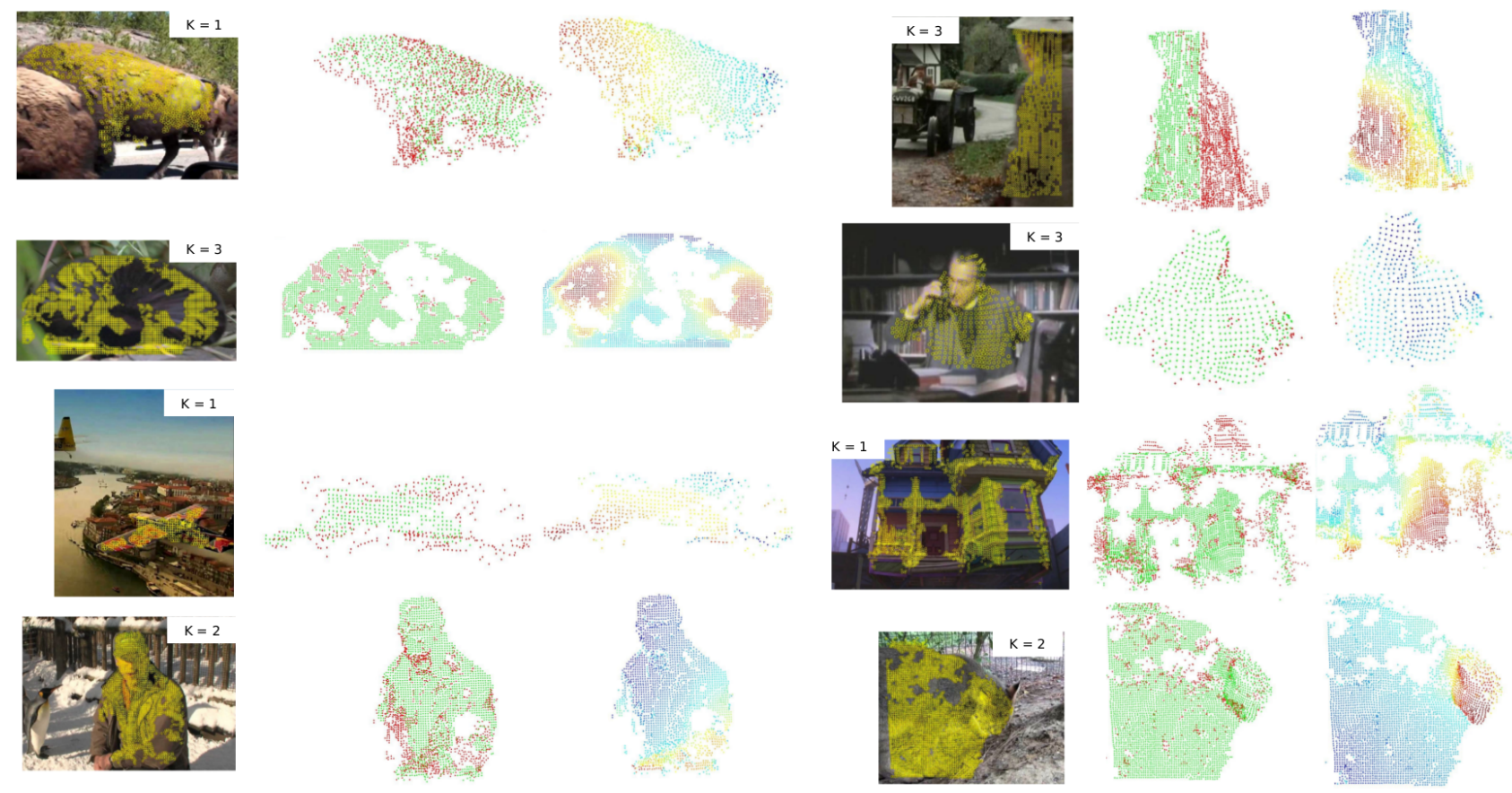
the pipeline illustrated in Figure 18, where we first detect and segment object instances (Section 6), and estimate their coarse pose (Gupta et al., 2015) to initialize a search over a small set of 3D models, their scales and precise placements. Figure 19 shows some sample results, where we can see that we can successfully address this problem in cluttered real world scenes.

Fig. 18. Pipeline to represent objects in RGB-D images with corresponding models from a 3D CAD model library.

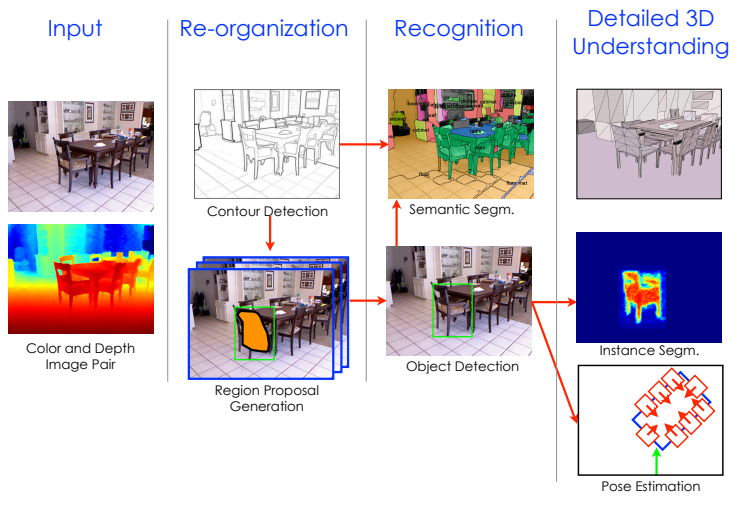

Fig. 19. Model Placement: Color image, placed models projected onto color image, depth map of rendered models (blue is closer, red is farther away).

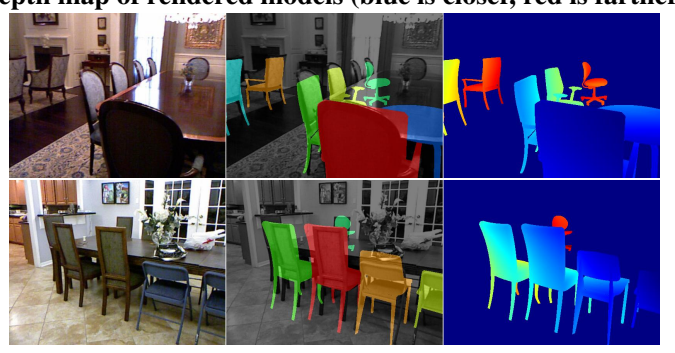

Analogous work for RGB images is presented by Kar et al. (2015). We first detect and approximately segment objects in images using the approach from (Hariharan et al., 2014) (Section 3). Then, as outlined in Section 4, for each detected object we estimate coarse pose for using the approach from (Tulsiani and Malik, 2015). We use the predicted object mask and coarse pose to fit the top down deformable shape models that were learned from data. Finally, we recover high frequency shape details using low level shading cues by conditioning the intrinsic image algorithm of Barron and Malik (2015) on our inferred coarse 3D shape. We present the first fully automatic reconstructions on the PASCAL VOC dataset (Everingham et al., 2010) and state-of-the-art object reconstruction as benchmarked on the PASCAL3D+ dataset (Xiang et al., 2014). Example results of reconstruction from a single image are shown in Figure 20.

Note that these two systems are merely examples of how the $3 R$ 's of reconstruction, reorganization and reconstruction can jointly work towards the grand goal of scene understanding. Probabilistic graphical models or neural network architectures with multiple loss terms are both frameworks which can support such synthesis efforts. Over the next few years, we may expect systems to keep improving in accuracy at benchmarks such as
MS COCO which emphasize detection and localization of individual objects, as well as other benchmarks which emphasize fidelity of geometric reconstruction.

But this is not all. Understanding a scene at the level of individual objects and their geometry is not the only goal of computer vision. Objects do not exist in isolation, they are in contextual relationships with each other and to humans and other agents that act in these environments. We can not claim to understand a scene fully until we have the ability to parse these relationships, make predictions about how the scene might evolve, and use our understanding of the scene to manipulate it or to navigate in it. Visual perception is not an end in itself, it connects to motor control as well as to general cognition. The progress that we have made so far provides an excellent substrate on which to build these connections.

\section{Acknowledgments}

This work was supported by ONR SMARTS MURI N0001409-1-1051, ONR MURI N00014-10-10933 and NSF Award IIS-1212798.

\section{References}

Aggarwal, J., Ryoo, M., 2011. Human activity analysis: A review. ACM Computing Surveys .

Akhter, I., Sheikh, Y., Khan, S., Kanade, T., 2011. Trajectory space: A dual representation for nonrigid structure from motion. IEEE Trans. Pattern Anal. Mach. Intell. 33

Arbeláez, P., Maire, M., Fowlkes, C., Malik, J., 2011. Contour detection and hierarchical image segmentation. TPAMI .

Arbeláez, P., Pont-Tuset, J., Barron, J., Marques, F., Malik, J., 2014. Multiscale combinatorial grouping, in: CVPR.

Barron, J.T., Malik, J., 2015. Shape, illumination, and reflectance from shading. IEEE Transactions on Pattern Analysis and Machine Intelligence .

Borenstein, E., Ullman, S., 2002. Class-specific, top-down segmentation, in: ECCV.

Bregler, C., Hertzmann, A., Biermann, H., 2000. Recovering non-rigid 3d shape from image streams, in: CVPR.

Brox, T., Malik, J., 2010. Object segmentation by long term analysis of point trajectories, in: ECCV.

Burer, S., Monteiro, R.D.C., 2005. Local minima and convergence in low-rank semidefinite programming. Math. Program. 103, 427-444. URL: http://dx.doi.org/10.1007/s10107-004-0564-1, doi:10. 1007/s10107-004-0564-1.

Burton, H.E., 1945. The optics of euclid. J. Opt. Soc. Am. 35, 357-357.

Cabral, R., de la Torre, F., Costeira, J., Bernardino, A., 2013. Unifying nuclear norm and bilinear factorization approaches for low-rank matrix decomposition, in: ICCV. doi:10.1109/ICCV. 2013.309.

Carreira, J., Sminchisescu, C., 2012. Cpmc: Automatic object segmentation using constrained parametric min-cuts. Pattern Analysis and Machine Intelligence, IEEE Transactions on 34, 1312-1328.

Carreira, J., Vicente, S., Agapito, L., Batista, J., 2015. Lifting object detection datasets into 3d. IEEE Transactions on Pattern Analysis and Machine Intelligence

Dai, Y., Li, H., He, M., 2012. A simple prior-free method for non-rigid structure-from-motion factorization, in: CVPR, pp. 2018-2025.

Dalal, N., Triggs, B., 2005. Histograms of oriented gradients for human detection, in: CVPR.

Deng, J., Dong, W., Socher, R., Li, L.J., Li, K., Fei-Fei, L., 2009. ImageNet: A large-scale hierarchical image database, in: CVPR.

Dollár, P., Zitnick, C.L., 2014. Fast edge detection using structured forests. CoRR abs/1406.5549.

Everingham, M., Van Gool, L., Williams, C.K.I., Winn, J., Zisserman, A., 2010. The Pascal Visual Object Classes (VOC) Challenge. IJCV 88. 

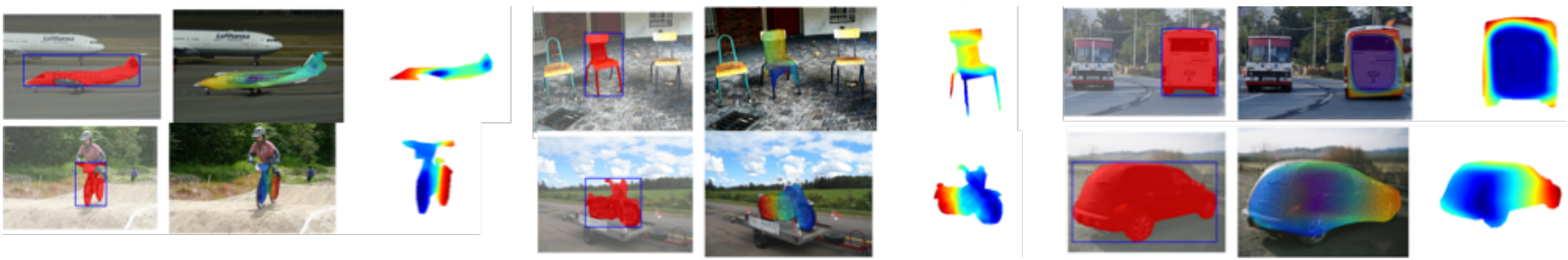

Fig. 20. Fully automatic reconstructions obtained from just a single image using our system described above. Each panel (from left to right) shows the image with automatic detection and segmentation, the 3D model inferred using our method 'replaced in place' and the final depth map obtained.

Farabet, C., Couprie, C., Najman, L., LeCun, Y., 2013. Learning hierarchical features for scene labeling. TPAMI 35.

Felzenszwalb, P., Girshick, R., McAllester, D., Ramanan, D., 2010. Object detection with discriminatively trained part based models. TPAMI .

Fidler, S., Mottaghi, R., Yuille, A., Urtasun, R., 2013. Bottom-up segmentation for top-down detection, in: CVPR.

Fragkiadaki, K., Salas, M., Arbelaez, P., Malik, J., 2014. Grouping-based lowrank trajectory completion and $3 \mathrm{~d}$ reconstruction, in: NIPS.

Galasso, F., Nagaraja, N.S., Cardenas, T.J., Brox, T., Schiele, B., 2013. A unified video segmentation benchmark: Annotation, metrics and analysis, in: ICCV.

Garg, R., Roussos, A., de Agapito, L., 2013. Dense variational reconstruction of non-rigid surfaces from monocular video, in: 2013 IEEE Conference on Computer Vision and Pattern Recognition, Portland, OR, USA, June 2328, 2013, pp. 1272-1279. URL: http://dx.doi.org/10.1109/CVPR. 2013.168, doi:10.1109/CVPR. 2013.168.

Ghodrati, A., Pedersoli, M., Tuytelaars, T., 2014. Is 2d information enough for viewpoint estimation, in: Proceedings of the British Machine Vision Conference. BMVA Press, p. 6.

Gibson, J.J., 1950. The perception of the visual world.

Girshick, R., 2015. Fast R-CNN. arXiv e-prints arXiv:1504.08083v1 [cs.CV].

Girshick, R., Donahue, J., Darrell, T., Malik, J., 2014. Rich feature hierarchies for accurate object detection and semantic segmentation, in: CVPR.

Girshick, R., Donahue, J., Darrell, T., Malik, J., 2015. Region-based convolutional networks for accurate object detection and segmentation. IEEE Transactions on Pattern Analysis and Machine Intelligence

Girshick, R., Felzenszwalb, P., McAllester, D., . Discriminatively trained deformable part models, release 5. http://www.cs.berkeley.edu/ rbg/ latent-v5/.

Gkioxari, G., Malik, J., 2015. Finding action tubes, in: CVPR.

Gu, C., Lim, J.J., Arbeláez, P., Malik, J., 2009. Recognition using regions, in: CVPR.

Gupta, S., Arbeláez, P., Girshick, R., Malik, J., 2014a. Indoor scene understanding with RGB-D images: Bottom-up segmentation, object detection and semantic segmentation. IJCV

Gupta, S., Arbeláez, P., Malik, J., 2013. Perceptual organization and recognition of indoor scenes from RGB-D images, in: CVPR

Gupta, S., Arbeláez, P.A., Girshick, R.B., Malik, J., 2015. Aligning 3D models to RGB-D images of cluttered scenes.

Gupta, S., Girshick, R., Arbeláez, P., Malik, J., 2014b. Learning rich features from RGB-D images for object detection and segmentation, in: ECCV.

Hariharan, B., Arbeláez, P., Girshick, R., Malik, J., 2014. Simultaneous detection and segmentation, in: European Conference on Computer Vision (ECCV).

Hariharan, B., Arbeláez, P., Girshick, R., Malik, J., 2015. Hypercolumns for object segmentation and fine-grained localization, in: Computer Vision and Pattern Recognition (CVPR).

Hartley, R., Vidal, R., 2008. Perspective nonrigid shape and motion recovery, in: Forsyth, D., Torr, P., Zisserman, A. (Eds.), Computer Vision ECCV 2008. Springer Berlin Heidelberg. volume 5302 of Lecture Notes in Computer Science, pp. 276-289. URL: http://dx.doi.org/10.1007/ 978-3-540-88682-2_22, doi:10.1007/978-3-540-88682-2_22.

He, K., Zhang, X., Ren, S., Sun, J., 2014. Spatial pyramid pooling in deep convolutional networks for visual recognition, in: ECCV.

Helmholtz, H.v., 1925. Physiological optics. Optical Society of America 3, 318.

Hoiem, D., Efros, A., Hebert, M., 2005. Geometric context from a single image, in: CVPR.

Hosang, J., Benenson, R., Dollár, P., Schiele, B., 2015. What makes for effec- tive detection proposals? arXiv e-prints arXiv:1502.05082v1 [cs.CV].

Hubel, D.H., Wiesel, T.N., 1962. Receptive fields, binocular interaction and functional architecture in the cat's visual cortex. The Journal of physiology 160.

Humayun, A., Li, F., Rehg, J.M., 2014. RIGOR: Reusing Inference in Graph Cuts for generating Object Regions, in: CVPR.

Jain, M., Gemert, J., Jegou, H., Bouthemy, P., Snoek, C.G.M., 2014. Action localization with tubelets from motion, in: CVPR.

Jhuang, H., Gall, J., Zuffi, S., Schmid, C., Black, M., 2013. Towards understanding action recognition, in: ICCV.

Kar, A., Tulsiani, S., Carreira, J., Malik, J., 2015. Category-specific object reconstruction from a single image, in: Computer Vision and Pattern Regognition (CVPR).

Krähenbühl, P., Koltun, V., 2014. Geodesic object proposals, in: ECCV.

Krizhevsky, A., Sutskever, I., Hinton, G.E., 2012. ImageNet classification with deep convolutional neural networks., in: NIPS.

Kruppa, E., 1913. Zur Ermittlung eines Objecktes aus zwei Perspektiven mit innerer Orientierung. Sitz.-Ber. Akad. Wiss., Wien, Math. Naturw., Kl. Abt. IIa 122, 1939-1948.

Lan, T., Wang, Y., Mori, G., 2011. Discriminative figure-centric models for joint action localization and recognition, in: ICCV.

LeCun, Y., Boser, B., Denker, J., Henderson, D., Howard, R., Hubbard, W., Jackel, L., 1989. Backpropagation applied to handwritten zip code recognition. Neural Comp.

Long, J., Schelhamer, E., Darrell, T., 2015. Fully convolutional networks for semantic segmentation, in: CVPR.

Lowe, D., 2004. Distinctive image features from scale-invariant keypoints. IJCV .

Maire, M., Yu, S.X., Perona, P., 2014. Reconstructive sparse code transfer for contour detection and semantic labeling, in: Asian Conference on Computer Vision (ACCV)

Malik, J., 2001. Visual grouping and object recognition, in: Image Analysis and Processing, 2001. Proceedings. 11th International Conference on, IEEE. pp. 612-621.

Marr, D., 1982. Vision: A computational approach.

Nandakumar, C., Torralba, A., Malik, J., 2011. How little do we need for 3-d shape perception? Perception-London 40, 257.

Pepik, B., Stark, M., Gehler, P., Schiele, B., 2012. Teaching 3d geometry to deformable part models, in: Computer Vision and Pattern Recognition (CVPR), 2012 IEEE Conference on, IEEE. pp. 3362-3369.

Pollefeys, M., Koch, R., Vergauwen, M., Gool, L.V., 1998. Metric 3d surface reconstruction from uncalibrated image sequences, in: 3D STRUCTURE FROM MULTIPLE IMAGES OF LARGE SCALE ENVIRONMENTS. LNCS SERIES, Springer-Verlag. pp. 138-153.

Poppe, R., 2010. A survey on vision-based human action recognition. Image Vision Computing

Ren, X., Malik, J., 2003. Learning a classification model for segmentation, in: Computer Vision, 2003. Proceedings. Ninth IEEE International Conference on, IEEE. pp. 10-17.

Roberts, L.G., 1963. Machine perception of three-dimensional solids. Technical Report 315. MIT Lincoln Laboratory.

Rodriguez, M.D., Ahmed, J., Shah, M., 2008. Action mach: a spatio-temporal maximum average correlation height filter for action recognition, in: CVPR.

Russell, B.C., Freeman, W.T., Efros, A.A., Sivic, J., Zisserman, A., 2006. Using multiple segmentations to discover objects and their extent in image collections, in: CVPR.

Sermanet, P., Eigen, D., Zhang, X., Mathieu, M., Fergus, R., LeCun, Y., 2014. OverFeat: Integrated Recognition, Localization and Detection using Convolutional Networks, in: ICLR. 
Sermanet, P., Kavukcuoglu, K., Chintala, S., LeCun, Y., 2013. Pedestrian detection with unsupervised multi-stage feature learning, in: CVPR.

Silberman, N., Hoiem, D., Kohli, P., Fergus, R., 2012. Indoor segmentation and support inference from RGBD images, in: ECCV.

Simonyan, K., Zisserman, A., 2014a. Two-stream convolutional networks for action recognition in videos, in: NIPS.

Simonyan, K., Zisserman, A., 2014b. Very deep convolutional networks for large-scale image recognition. arXiv preprint arXiv: 1409.1556.

Tian, Y., Sukthankar, R., Shah, M., 2013. Spatiotemporal deformable part models for action detection, in: CVPR.

Tighe, J., Niethammer, M., Lazebnik, S., 2014. Scene parsing with object instances and occlusion handling, in: CVPR.

Toh, K.C., Yun, S., 2010. An accelerated proximal gradient algorithm for nuclear norm regularized linear least squares problems. Pacific Journal of Optimization .

Tomasi, C., Kanade, T., 1991. shape and motion from image streams: a factorization method. Technical Report. IJCV.

Torresani, L., Hertzmann, A., Bregler., C., 2008. Non-rigid structure-frommotion: Estimating shape and motion with hierarchical priors. IEEE Transactions on Pattern Analysis and Machine Intelligence.

Tulsiani, S., Malik, J., 2015. Viewpoints and keypoints, in: Computer Vision and Pattern Regognition (CVPR).

Uijlings, J., van de Sande, K., Gevers, T., Smeulders, A., 2013. Selective search for object recognition. IJCV .

Vaillant, R., Monrocq, C., LeCun, Y., 1994. Original approach for the localisation of objects in images. IEE Proc on Vision, Image, and Signal Processing

Viola, P., Jones, M., 2001. Rapid object detection using a boosted cascade of simple features, in: CVPR.

Wang, H., Schmid, C., 2013. Action recognition with improved trajectories, in: ICCV.

Wang, L., Qiao, Y., Tang, X., 2014. Video action detection with relational dynamic-poselets, in: ECCV.

Wang, X., Yang, M., Zhu, S., Lin, Y., 2013. Regionlets for generic object detection, in: ICCV.

Weinland, D., Ronfard, R., Boyer, E., 2011. A survey of vision-based methods for action representation, segmentation and recognition. Computer Vision and Image Understanding

Wertheimer, M., 1923. Laws of organization in perceptual forms. A Source Book of Gestalt Psychology .

Xiang, Y., Mottaghi, R., Savarese, S., 2014. Beyond pascal: A benchmark for $3 \mathrm{~d}$ object detection in the wild, in: Applications of Computer Vision (WACV), 2014 IEEE Winter Conference on, IEEE. pp. 75-82.

Yang, Y., Hallman, S., Ramanan, D., Fowlkes, C.C., 2012. Layered object models for image segmentation. TPAMI 34.

Zitnick, C.L., Dollár, P., 2014. Edge boxes: Locating object proposals from edges, in: ECCV. 
Related material (NOT FOR PUBLICATION IN THIS PAPER)
Click here to download Related material (NOT FOR PUBLICATION IN THIS PAPER): BharathECCV2014.pdf Cha

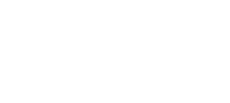
(1) (1) 
Related material (NOT FOR PUBLICATION IN THIS PAPER)

Click here to download Related material (NOT FOR PUBLICATION IN THIS PAPER): Girshick_Rich_Feature_Hierarchies_2014_C 
Related material (NOT FOR PUBLICATION IN THIS PAPER)
Click here to download Related material (NOT FOR PUBLIC Click here to download Related material (NOT FOR PUBLICATION IN THIS PAPER): Gkioxari_Finding_Action_Tubes_2015_CVPF kioxar Finding 
Related material (NOT FOR PUBLICATION IN THIS PAPER)
Click here to download Related material (NOT FOR PUBLICA

Click here to download Related material (NOT FOR PUBLICATION IN THIS PAPER): Hariharan_Hypercolumns_for_Object_2015_ (1) 
Related material (NOT FOR PUBLICATION IN THIS PAPER)
Click here to download Related material (NOT FOR PUBLIC

Click here to download Related material (NOT FOR PUBLICATION IN THIS PAPER): Kar_Category-Specific_Object_Reconstructi 
Related material (NOT FOR PUBLICATION IN THIS PAPER)
Click here to download Related material (NOT FOR PUBLICATION IN THIS PAPER): NIPS2014_NRSFM.pdf (

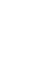



(

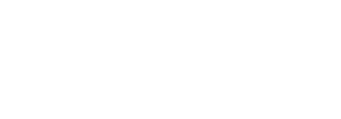


Related material (NOT FOR PUBLICATION IN THIS PAPER)
Click here to download Related material (NOT FOR PUBLIC

Click here to download Related material (NOT FOR PUBLICATION IN THIS PAPER): Tulsiani_Viewpoints_and_Keypoints_2015_

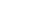

Click here to download Related material (NOT FOR PUBLICA

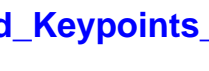


LaTeX Source Files
Click here to download LaTeX Source Files: pr-3r.zip

LaTeX Source Files
Click here to download LaTeX Source Files: pr-3r.zip

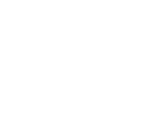

(n)
(2)

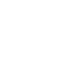

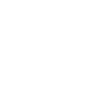

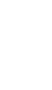
.

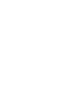

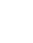
.

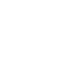

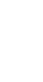
.

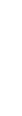
.

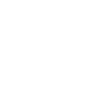

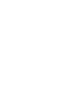

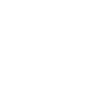

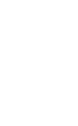

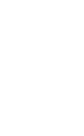

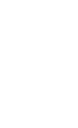

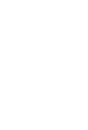

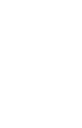

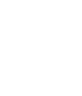

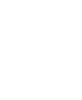

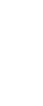

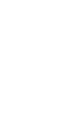

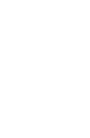

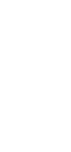
更

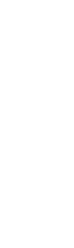
更

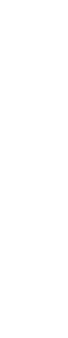

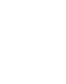

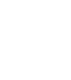

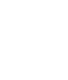

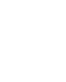

